\title{
Evaluation of the Benefits of Implementing a Smart Pedestrian Network System
}

https://doi.org/10.1515/comp-2020-0127

Received Mar 31, 2020; accepted Jul 08, 2020

\begin{abstract}
Information and Communication Technology (ICT), and recent advancements in Computer Science can serve as a catalyst for promoting sustainable means of transport. Through ICT applications, active mobility can be promoted and established as a viable transport mode. This can be achieved by providing relevant information for fostering social capital and promoting physical activity, thus contributing to a higher quality of life. Further, active mobility can greatly contribute to reducing air pollution and improving health status. For this purpose, the implementation of a Smart Pedestrian Network (SPN) information system is proposed. Such an implementation requires the collaboration of various stakeholders including the public, local authorities and local businesses. To convince stakeholders of the viability of implementing SPN, the benefits of active mobility should be clear. This paper proposes a framework to quantify active mobility benefits so that stakeholders can assess the investment that can be realized from implementing SPN. The proposed framework makes use of quantifying benefits in various market conditions. The benefits are shown to be significant and very much in favor of investing in technology and implementing the envisioned SPN system.
\end{abstract}

Keywords: Information and Communication Technology (ICT), Software development, Sustainable mobility, Smart Pedestrian Network

\footnotetext{
^Corresponding Author: George Papageorgiou: E.U.C.
Research Center, European University Cyprus; Email:

^Corresponding Author: George Papageorgiou: E.U.C.
Research Center, European University Cyprus; Email: g.papageorgiou@euc.ac.cy Anastasia Ioannou: European University Cyprus; Email: ai185155@students.euc.ac.cy

Athanasios Maimaris: SYSTEMA Research Center, European University Cyprus; Email: a.maimaris@cycollege.ac.cy

Alexander Ness: University of Oslo, Institute of Economics; Email: alexnn@student.sv.uio.no
}

\section{Introduction}

Advancements in Computer Science and Information and Communication Technology (ICT), present a great opportunity for promoting active mobility urban development strategies. Despite the opportunity it should be noted that in recent years ICT applications were mainly used to facilitate motorized vehicles by optimizing traffic flows via Intelligent Transportation Systems (ITS) techniques [16]. Unfortunately, this situation promotes vehicle traffic, which creates more air pollution with negative effects on human health and negative consequences for the natural environment. Specifically, this paper investigates the benefits accrued from the use of ICT to promote active mobility via implementing a Smart Pedestrian Network (SPN) information system in municipalities around Europe.

Active means of transport must be encouraged if we are to address major environmental problems such as pollution and climate change, but also, improve well being and quality of life. With an estimated half a million [3] people in Europe alone dying from diseases triggered by air pollution, it is imperative that local authorities take drastic measures to ensure that pollutants stemming from vehicle use are reduced to zero. Active mobility such as walking can offer multiple benefits to governments, society, the environment and individuals. Currently, air pollution is costing the global economy 4.5 trillion USD annually [22]. A large proportion of this is due to health issues arising from air pollution. By encouraging the substitution of vehicle use with active means of transport, governments and people, can expect to see a significant reduction in healthcare costs. Furthermore, illnesses and premature deaths caused by air pollution impede economic activity and development [10]. Therefore, economic performance also benefits from increased walking levels, if it means that fewer people will be sick and thus absent from work. In other words, active mobility can increase labor productivity. Here ICT applications informing people about the multiple benefits from active mobility can act as a catalyst for cultivating an active mobility culture.

Further, the contaminated and heavily polluted oceans are also a major issue. Organizations, the media and en-

əopen Access. ( $2021 \mathrm{G}$. Papageorgiou et al., published by De Gruyter. 
vironmental groups are constantly reminding people and governments of the detrimental effects that human activity is having not only on human life, but also on the animal kingdom and the ecosystem as well [15]. Habitats of the ecosystem are constantly under threat and soon, many species are predicted to be extinct. If we do not immediately attend to the issue of climate change, the planet as we know it, will no longer be sustainable. Promoting walking and more generally active mobility instead of driving, can greatly help maintain cleaner oceans and ecosystems.

Active mobility can also help individuals build stronger trust and bonds between family, friends and neighbors [20] The simple act of walking - an action that is free and readily available to most people - has also shown to improve peoples' social, psychological, emotional and mental well being [2]. This alone exemplifies the myriad of benefits that governments, municipalities, the economy, the environment and individuals can gain from walking and active mobility. The digitally interconnected world we live in, provides the necessary prerequisites for developing software applications so that the active mobility transport mode becomes safe, convenient, enjoyable and attractive.

A lot more can be achieved if active mobility implementation programs involve ICT applications. Unfortunately, so far, interventions to promote active mobility have not utilized the power of ICT and have failed to properly address climate change and provide adequate long term solutions. In the past, governments have tried to improve road networks to reduce congestion in the hopes of reducing carbon emissions. However, this solution resulted in unwelcome repercussions; rather than reducing carbon emissions, such policies of "building roads" only increase congestion due to the fact that more people are now encouraged to drive. If we are to help attenuate the traffic congestion problem, and consequently air pollution, we must look to more sustainable means of transport supported by ICT.

This is why we posit the implementation of a Smart Pedestrian Network (SPN) information system that will be geared towards encouraging people to walk more, by providing them with suitable walking routes, customized on their particular needs. For instance, elderly people may require special amenities such as benches to rest, or water fountains along their walking routes to keep hydrated. Tourists may wish to walk along a path that entails better sightseeing. SPN takes the form of a smartphone application to provide users with the most appropriate walking routes to satisfy such needs. In addition, SPN could provide rewards and incentives for people who walk more. For instance, by collaborating with cafes, restaurants and other businesses, we can provide special offers to SPN users who walk a specified number of steps.

Of great importance is to be able to evaluate the benefits of walking as well as the potential and profitability of implementing SPN, taking into account costs, revenues and other benefits - these must be quantified and monetized. It is vital to quantify such factors to assess the magnitude of benefits accrued from walking. In turn, information about the benefits can be provided to people by the ICT application. In this way, we could motivate people to walk more and convince stakeholders to support SPN implementation as it would represent a worthwhile investment.

In this paper, a framework is presented which serves as a guidance on how to estimate the benefits of implementing SPN. These benefits include reduced healthcare costs, reduced fuel costs and reduced monthly expenses. To be able to showcase this, we work on a hypothetical scenario where SPN is implemented in an urban region within Europe. In this way, an illustrative example is provided and evaluated on its further application prospects in Europe. The framework makes use of discounted cash flow methods. This is important for investigating the long term impacts the SPN will have on the natural environment, the economy, governments, ecosystems and individuals. This information would be very important to policymakers and other stakeholders to invest in this ICT application.

The paper is structured as follows. In section 2, the literature review is presented whereby studies evaluating the benefits of active mobility and walking are discussed. Furthermore, current global issues caused by climate change and vehicle use are also highlighted. Section 3 discusses the development and implementation of SPN. A specific scenario is set up, whereby SPN aims to target a certain market share within Europe, and how given this, we can calculate all possible benefits. Further, in section 3, we also examine all possible monthly costs of running the SPN project, and how SPN will obtain its monthly revenues. Finally, conclusions are given regarding the viability of implementing the Smart Pedestrian Network concept and the many benefits accrued.

\section{Literature Review}

Health benefits attributed to walking have been the element of enquiry in contemporary research. Many studies have found that walking 10,000 steps per day [23] can help individuals suffering from high blood pressure, improve body composition and serum lipid levels. Walking 10,000 steps per day offers benefits similar to other moderate forms 
of exercise that people may carry out. Therefore, walking can provide a cheaper and easier alternative to other forms of exercise. Such information is important to be given to people and can be easily provided by an ICT application.

Obesity is currently a severe health issue that $50 \%$ of men and women in Europe are facing [25]. This not only results in poor quality of life, but possible greater healthcare costs for individuals and governments as well as premature deaths. Obesity has also been linked to increased sedentary behavior and a decline in walking levels. But many studies have found that active mobility like walking can indeed not only help prevent weight gain but also reduce obesity. Senauer and Gemma [21] conducted a study comparing psychical activity of Japanese and Americans. A high percentage (67\%) of Americans were classified as obese compared to only $25 \%$ of Japanese. When examined why Japan has one of the lowest obesity rates in the world, an important finding was uncovered. Japanese people are more physically active than Americans; but this was not due to engaging in more planned physical activity such as going to the gym or swimming. It was attributed to Japanese people engaging more in active mobility. Senauer and Gemma [21] found that Americans used their vehicles to a much greater degree than Japanese people and suggested that the American government should encourage the public to walk more and use their cars less. Furthermore, Harvard Medical School [9] found that walking briskly for around 1 hour per day reduces the effect of these obesity-promoting genes by $50 \%$. In addition, it was also found that walking can promote other health benefits including reduced cancer risk, joint pain relief and a healthier immune system.

Hill [11], found that walking 30 minutes per day can aid people with diabetes. Currently, 60 million people in Europe have been diagnosed with diabetes [24]. Such discoveries therefore also denote how walking can help diabetics. Hill [11] also cites how using technological equipment such as counting the number of steps via a smartphone to encourage people to walk more can be a great incentive. Implementing SPN could further gamify the experience of walking. Today, counting number of steps is a standard feature of every smartphone. In the study, Hill [11] looked at diabetics who all underwent counselling that aimed to increase their physical activity. Participating patients were given specific targets that was aimed at increasing their walking levels over time. Hill [11] concluded that even those who did not manage to reach their targets saw an improvement in their health, while those who achieved their targets saw a much larger development in their health.

In contrast to what the majority of people may believe about driving to places, vehicle use can actually be an inefficient, time-consuming and costly means of travel [13]. It has actually been found to reduce punctuality of workers. This is all due to the increased probability of a car breaking down, road accidents and traffic congestion. All these factors hinder a person's productivity and efficiency both in their professional capacity and their personal lives. Furthermore, road accidents and cars breaking down also produce negative knock on effects for other individuals who may be travelling along the same route. Contrary to this, active mobility can reduce work absenteeism, increase energy levels and ensure that people reach their destinations in time. Such consequences will thus also positively impact one's career development and progression.

As already mentioned, walking can also contribute to increasing social capital and help build better relationships amongst friends, families and neighbors. Kim and Yang [12] undertook a study to ascertain a possible link between social capital and walking levels. To achieve this, data was obtained from 591 individuals in Seoul, regarding the reasons for walking and participants opinions on the possible gains they got from their walking experience. The most important finding from this study was that strong levels of trust was seen between neighbors who frequently walked together for leisure compared to those who did not. Hence, Kim and Yang [12] deduced that walking can aid in reinstating social functions within neighborhoods.

It is important to grasp how individuals perceive sustainability, and in what way walking can impact social capital. Rogers et al. [20] interviewed key informants, municipal decision makers and neighborhood leaders in New Hampshire, Manchester and Portsmouth in the United States. The appropriate demographic data was obtained on neighborhoods in these regions and a social capital survey conceived by [8] was given out to participants. The study is extremely relevant for the purpose of our research as it deals with directly asking participants what motivates them to walk and how high are the trust levels within their communities. The most important factors that motivate people to walk are safety, the provision of sidewalks and lighting. This is also in line with findings from previous research [17] indicating that safety and pedestrian network conditions are crucial determinants of walking. These results are important for policy formulation and implementation. To ensure the right policies are enacted, peoples' mindsets and cognitive behavior must be accounted for. By doing so, we can hope to give incentives to people to walk more by providing them with the right conditions such as safe walking routes and adequate infrastructure.

Ball et al. [1] investigated the relationship between aesthetically appealing environments, convenience of walking, the presence of a companion when walking and walking levels. The study did this accounting for differences be- 
tween men and women and reported mental and physical health. This was carried out by collecting and analyzing cross sectional self-report data from a survey that consisted of 3,392 Australian adults. Further, Ball et al. [1] found that men and women who found their environment less aesthetically appealing or less convenient had a lower probability of walking for exercise or recreation compared to those who found their environment more aesthetically pleasing or convenient. Furthermore, both men and women who had no companion to walk with along their walking routes including pets, were also less likely to walk for exercise or recreation. This observation was particularly more so for women than for men. This could also be due to safety reasons. Both men and women who were classified as having good or poor physical and mental health all felt that the aesthetic appeal of the environment and companionship played a prominent role in their decision to walk or not for exercise or recreation.

It was therefore concluded that convenience, environmental aesthetics and companionship are all significant determinants for walking and thus should be strongly considered when formulating policies. Indeed, if policy makers are to promote walking, they should to focus on ensuring that walking paths are smooth and convenient so that people can reach their destinations quickly, comfortably and safely. Furthermore, governments and municipalities should also invest in creating beautiful parks, planting trees and providing amenities such as public toilets, water fountains and benches that will all contribute to a more aesthetically appealing environment.

As already suggested, technological equipment can help in encouraging walking amongst the public. Information and Communication Technology (ICT) can provide incentives for people to track their progress through counting the number of steps and gamifying the experience of walking, monitoring heart rate and calories burnt [4]. The SPN system [19] incorporates such features so that users will be more inclined to walk and thus improve health and sustainable development. In addition, SPN may have a way of informing users of the $\mathrm{CO}_{2}$ emissions that have been reduced as a result of their walking journeys. By entailing such features, SPN can be an important contributor to combating climate change. Papageorgiou et al. [18], discuss how SPN will show tailored walking routes to users and enable them to enjoy their walks with family and friends as well as collect rewards for engaging in active mobility. Moreover, SPN acts as a channel by which SPN users can contact and inform governments and municipalities about problems in current pedestrian conditions.

What becomes very important is to be able to justify investing in the implementation of a Smart Pedestrian Net- work Information system. Such a justification could be carried out by quantifying the benefits accrued from implementing SPN and promoting active mobility. In the next section, a framework is presented for evaluating such benefits in a quantified manner. Note that the proposed framework could be utilised by other similar projects in order to prove their viability. In this way, a significant gap is fulfilled in the current literature.

\section{A Framework for Evaluating the Benefits of Active Mobility}

As shown above, information about the benefits from implementing an ICT application to promote walking, is highly important. Therefore, somehow this information of quantifying the benefits should be accounted for in the software development process. This section presents a framework for evaluating benefits to be included in the SPN system development. The proposed framework is shown in Fig. 1. Note that once the system is designed, developed and tested, information about the benefits should be provided to the end users. This is imperative so that end users are convinced to use the system as well as stakeholders to invest in further system development. In order to provide information about the benefits, quantification should be carried out, which is presented in this section.

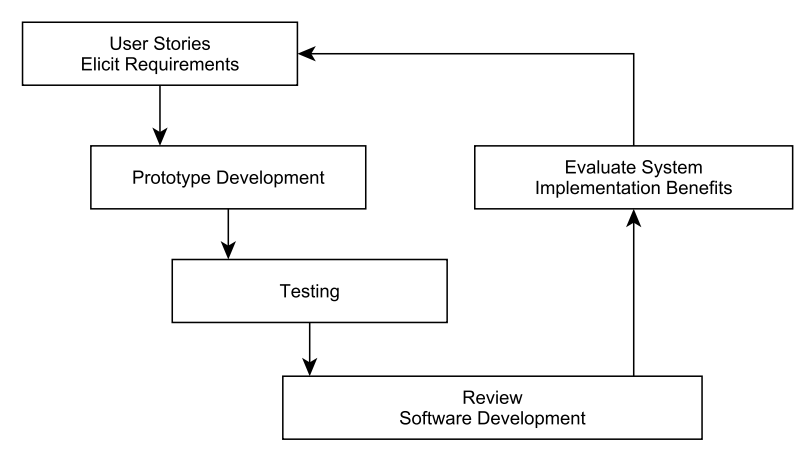

Figure 1: Proposed Software Development Method, Accounting for Implementation Benefits.

Consider a case where the development team of SPN wants to target a specific share of the market denoted by $x$, within a given region. Let $\alpha$ be the population that lies within this specific region. Therefore, the targeted market denoted by $y$ within the given region would be:

$$
y=x^{\star} \alpha
$$


Based on the literature review, it was revealed that walking can contribute a lot to lowering healthcare costs associated with obesity, cardiovascular diseases (CVDs) such as coronary artery disease, diabetes and deaths attributed to pollution. The number of people within the given population of the region $\alpha$ that suffer from obesity, coronary artery (CA) disease, diabetes and deaths attributed to pollution are characterized by $\beta, \gamma, \phi$ and $\lambda$ respectively. To calculate the number of individuals suffering from obesity, CVDs, diabetes and deaths attributed to pollution within the target audience $y$ which will be denoted by $r, q, s$ and $z$, we estimate the following:

$$
\begin{aligned}
& r=\left(y \star \frac{\beta}{\alpha}\right) \\
& q=\left(y \star \frac{\gamma}{\alpha}\right) \\
& s=\left(y \star \frac{\phi}{\alpha}\right) \\
& z=\left(y \star \frac{\lambda}{\alpha}\right)
\end{aligned}
$$

Data on the amount on healthcare costs pertaining to pollution within the region can be obtained. It is estimated that $75 \%$ of air pollution is caused by vehicle use which has significant negative effects on peoples' health [6]. Therefore, $75 \%$ of healthcare costs associated with air pollution can be reduced by increasing walking levels. Let these healthcare costs be denoted by $h$. To calculate the per capita healthcare costs $c$, the healthcare costs $h$ should be divided by the total population $\alpha$. Based on a study conducted by the WHO [26], the healthcare costs $h$ associated with air pollution, represents $4 \%$ of GDP as shown in the equation below:

$$
€ c=\left(0.04 * \frac{G D P}{\alpha}\right)
$$

Each person in the target region on average accounts for $€ c$ in healthcare costs owed to pollution.

Let it be further assumed that implementing SPN can influence people to walk a further 20,000 steps on average per week - which corresponds to $18 \mathrm{~km} /$ week. Therefore, each of these individuals within the target audience who suffer from obesity, coronary artery disease, diabetes or carry the risk of death, can reduce this healthcare costs by a specified amount. A study [7] have found that walking can reduce cholesterol levels by $7 \%$ thereby reducing obesity, CA disease by $9 \%$ diabetes by $12 \%$ and deaths by $27 \%$. Therefore, estimated reduced healthcare costs of obesity, CVDs, diabetes and deaths in the target audience will be:

$$
\text { ObesityInd }=(c) \text { * (Obesity) } *(0.07)
$$

$$
\begin{gathered}
\text { CAdiseasInd }=(c) \star(\text { CAdisease }) \star(0.09) \\
\text { DiabetesInd }=(c) \star(\text { Diabetes }) \star(0.12) \\
\text { DeathsInd }=(c) \star(\text { Deaths }) \star(0.27)
\end{gathered}
$$

Where ObesityInd, CAdiseaseInd, DiabetesInd and DeathsInd are the healthcare cost savings realized from obesity, CVDs, diabetes, and death respectively if people who fall within these categories walk 20,000 more steps per week. The total cost savings in healthcare therefore is the total sum of these four variables:

$$
\begin{array}{r}
\text { HealthcareCostSavings }=\text { ObesityInd } \\
+ \text { CAdiseaseInd } \\
+ \text { DiabetesInd } \\
+ \text { DeathsInd }
\end{array}
$$

In a similar fashion, we can estimate the possible cost savings that individuals make from reduced vehicle use. More specifically, if individuals walk more and use their cars less, they will be able to reduce fuel costs. On average in Europe, it was estimated that fuel prices are $€ 1.34 /$ liter [5] and, on average, cars within the European Union travel 12,000 kilometers per year [14]. Since there are 52 weeks in a calendar year, this equates to $230 \mathrm{~km} /$ week. A fuel efficient car on average consumes 1 liter of fuel for every $16 \mathrm{~km}$. Therefore, if an individual walks $18 \mathrm{~km}$ per week, they will save approximately $€ 1.50$ on weekly fuel. This can then be aggregated for all individuals using SPN so that the total amount saved on petrol in this region is $y \star € 1.50$. This can then be multiplied by 52 weeks, to estimate cost savings made on petrol per year.

SPN would also provide users with incentives to motivate them to walk. This can be in the form of free/discounted meals/drinks, discounted cinema tickets or even cash rewards. Whatever form the discounted/free item takes, this implies that an individual will have more money each month to spend elsewhere. Let it be assumed that each SPN user can save 5\% of their monthly spending by redeeming rewards. Obtaining data on the average monthly spending in this specified region, will enable us to calculate the monthly savings accrued from SPN rewards and incentives per capita and for the entire target audience. If the monthly spending is $€ b$, then each SPN user can save $€ b^{\star} 0.05$ every month to walk around 80,000 steps.

If the market share of SPN is to grow each year, the realized benefits will grow as well. To calculate the benefits, an appropriate discounted cash flow method should be applied. The framework makes use of Net Present Value 
$(N P V)$ and suggests employing a constant discount rate that will be denoted by $r$. For a given period $T$ that denotes the number of years, $r$ can be applied to estimate the NPV of each of these benefits. Therefore, $N P V$ is such that:

$$
\begin{aligned}
N P V & =\frac{\text { Benefits }}{1+r}+\frac{\text { Benefits }}{(1+r)^{2}}+\frac{\text { Benefits }}{(1+r)^{3}} \ldots \\
& +\frac{\text { Benefits }}{(1+r)^{T}}
\end{aligned}
$$

Therefore, $N P V$ can be written as:

$$
N P V=\sum_{T=0}^{n} \frac{\text { Benefits }}{(1+r)^{T}}
$$

The income streams of SPN can be calculated in a similar manner. SPN will be free to download with a Freemium and Premium option available. The Premium will be useful for those wishing to obtain more advanced SPN features such as expert advice from nutritionists or greater rewards from walking. SPN Premium will cost $€ 1 /$ month. Therefore, if it is assumed that $10 \%$ of SPN users will purchase the Premium version, this will amount to:

$$
\text { MonthlyPremiumIncome }=€ 1 \text { * } 0.1 \text { * } y
$$

Also, SPN will be open to donations. For instance, donations can be made of a specific amount, say $€ 0.50$ for every SPN user. This can amount to $€ 0.50^{\star} y$ per year. Similarly, sponsorship are another way that the SPN project can raise funds. We can collaborate with firms interested in sustainability to promote their businesses in return for extra funding. So, companies might be especially interested in sponsoring SPN. For instance, $€ 1$ every year for every SPN user can be given which will amount to $€ 1$ * $y$ per year.

Furthermore, advertising will be another main source of SPN income. This will be via push button profits. For every advert that is clicked on by SPN users, SPN will gain $€ 0.10$. Let it be assumed that $5 \%$ of the targeted audience will click on adverts every day. This will amount to $€ 0.10 * 0.05 * y$. Multiplied by 365 days, and divided by 12 to calculate income from adverts for a calendar month, we obtain:

$$
\begin{aligned}
& \text { MonthlyAdvertisementIncome }= \\
& \frac{(0.10 \star 0.05 * y)(365)}{12}
\end{aligned}
$$

An important source of income for SPN will arise from insurance partner companies or medical funds as these companies and funds will realize cost savings in terms of lower healthcare costs. As a result, a percentage of these cost savings should be paid back to the SPN users, with a percentage given to SPN. These amounts can be calculated based on the number of steps walked by SPN users. A viable proposition would be that $25 \%$ of cost savings will be kept by insurance companies, $25 \%$ will be given to SPN and $50 \%$ will be given to SPN users. Let us assume that $30 \%$ of insurance companies and/or medical funds within the specified region cooperate with SPN. We can calculate the income of SPN from healthcare cost savings per step for every year as follows:

$$
\text { HealthcareIncome }=0.25 \star 0.30 \star \text { HealthcareCost }
$$

Further, support from municipalities and governments will also provide additional sources of income for SPN. As already seen, the cost savings of such an application can be significant. Local authorities can use these funds towards other government projects. Such government projects can include supporting SPN as a means of promoting sustainable development. A small fraction of the cost savings due to increased levels of walking can be given to SPN. NPV can also be calculated for income streams and costs of SPN, by applying the constant discount factor $r$ such that the total NPV of the SPN project will be:

$$
\begin{aligned}
\text { TotalNPV } & =\text { NPVofIncomeStreams } \\
& - \text { NPVofCosts } \\
& =\sum_{T=0}^{n} \frac{\text { Income }}{(1+r)^{T}}-\sum_{T=0}^{n} \frac{\text { Costs }}{(1+r)^{T}}
\end{aligned}
$$

The costs of implementing SPN per city are relatively low, since the software of SPN has already been developed via a European funded project (sunk cost). It is estimated that the monthly cost for the server is $€ 200 /$ month while the cost of supporting SPN via Apple and Google app stores is $€ 90 /$ month. In addition, we will have personnel, marketing and advertising costs, as well as overheads. Running a hypothetical scenario using computer simulation, it became apparent that SPN is a worthwhile investment. Comparing the costs with the revenue streams, it is clear that implementing the SPN project is indeed a profitable venture.

Note that there are several other benefits that have not been accounted for in the proposed framework such as higher productivity, utility from walking, social capital levels, and reduced building maintenance costs from air pollution. If these were also accounted for, definitely, benefits would be higher. 


\section{Conclusion}

Prior to investing in Information and Communication Technology (ICT) system solutions to promote sustainability, it is important to understand the benefits to be realized by governments, individuals, the economy, the natural environment. To provide clarity, quantification of such benefits becomes essential. However, the process of quantification is far from simple, especially for the case of investing in ICT to promote active mobility. For instance, when a person walks, they obtain a certain level of satisfaction or utility. But how can we measure accurately this level of utility? There is no consensus on an appropriate method to calculate utility. Often, surveys are given out to people for them to rank or rate their preferences. Furthermore, happiness indexes are used to be able to gather information on utility levels. However, this implies relying on information delivered by individuals themselves regarding their utility evaluation. But often, people incorrectly estimate their true utility levels. People have a tendency to also overestimate the utility from tangible items such as discounted/free goods they can get from using SPN. In contrast, people tend to underestimate the utility they obtain from non-tangible items. In the case of the SPN system, this can involve their increased social capital levels as a utility.

It is evident that placing a monetary value on something that has no intrinsic value is far from simple. On the other hand, by gathering as much information as possible from people and perhaps implementing other methods such as looking at a person's willingness to pay for something, may be a good first step to build a solid grasp on how people view active mobility.

In this paper, we evaluate benefits of implementing a Smart Pedestrian Network (SPN) information system which promotes walking. This is achieved by developing a framework for quantifying the main benefits that arise from using the SPN system and thereby increasing walking levels. It can be clearly seen that by implementing SPN creates benefits the natural environment, people, governments, the economy and ecosystems. Increased walking can reduce the likelihood of developing certain health issues including diabetes, obesity, cardiovascular diseases and even premature death. Consequently, it can help to significantly reduce healthcare costs. In return, this not only helps stimulate greater economic activity and development by promoting greater labor productivity, but it also enables governments and people to save on healthcare costs and allocate funds to other projects or needs they may have. Similarly, if people save on fuel costs, they will see an increase in their real income and their monthly budgets.
Implementing SPN would also encourage people to walk more by providing incentives in the form of rewards. Therefore, people can also reduce monthly expenses by walking as they receive free/discounted goods, vouchers for shopping and even cash rewards. Calculating the costs and income streams showed that SPN has great viability as the revenue streams can be relatively large, and thus would be a worthy investment. All costs and income streams can be calculated in the proposed framework using discounted cash flow methods. It is clear that implementing sustainable Information and Communication Technology (ICT) solutions such as the case of the SPN system development produces long term benefits that will help contribute to a more sustainable planet. It is vital to incorporate this significant stage of quantifying the benefits in any software development process.

Acknowledgement: The research presented in this paper is co-funded by the Republic of Cyprus and the European Regional Development Fund as part of ERANET Cofund Smart Urban Futures (ENSUF) Joint Programming Initiative (JPI) Urban Europe, through the Research Promotion Foundation, protocol no. KOINA/ПKП URBAN EUROPE/1215/11. This framework is supported by the European Commission and funded under the HORIZON 2020 ERANET Co-fund scheme

\section{References}

[1] Ball K., Bauman A., Leslie E., Owen N., Perceived Environmental Aesthetics and Convenience and Company Are Associated with Walking for Exercise among Australian Adults, Preventive Medicine, 2001, 33(5), 434 - 440, ISSN 0091-7435.

[2] Barton J., Hine R., Pretty J., The health benefits of walking in greenspaces of high natural and heritage value, Journal of Integrative Environmental Sciences, 2009, 6(4), 261-278.

[3] Berthier D., Air pollution kills more than 500,000 Europeans each year, 2018, https://www.europeandatajournalism.eu/ News/Data-news/Air-pollution-kills-more-than-500-000-Euro peans-each-year, Accessed: 2019-09-01.

[4] Conticelli E., Maimaris A., Papageorgiou G., Tondelli S., Planning and Designing Walkable Cities: A Smart Approach, 2018, 251269, Springer International Publishing, Cham, ISBN 978-3-31977682-8.

[5] Drive Alive, Fuel prices in Europe: the cost of petrol and diesel in Europe. All petrol and diesel prices in Euros at July 18th 2019, 2019, https://www.drive-alive.co.uk/fuel_prices_europe.html, Accessed: 2019-09-01.

[6] European Environment Agency, Premature deaths attributable to air pollution, 2016, https://www.eea.europa.eu/media/ newsreleases/many-europeans-still-exposed-to-air-pollution2015/premature-deaths-attributable-to-air-pollution, Accessed: 2019-09-01. 
[7] Glatter R., Brisk Walking or Running: Both Reduce Risk for Heart Disease, 2013, https://www.forbes.com/sites/robertglatter/2 013/04/05/brisk-walking-or-running-both-reduce-risk-for-hea rt-disease/\#5281bd61461e.

[8] Harvard Kennedy School, SocialCapitalShort-FormSurvey, 2018, http://www.hks.harvard.edu/programs/saguaro/measureme nt\#shortform, Accessed: 2019-05-01.

[9] Harvard Medical School, 5 surprising benefits of walking, 2019, https://www.health.harvard.edu/staying-healthy/5-surprisingbenefits-of-walking, Accessed: 2019-09-01.

[10] He J., Liu H., Salvo A., Severe Air Pollution and Labor Productivity: Evidence from Industrial Towns in China, American Economic Journal: Applied Economics, 2019, 11(1), 173-201.

[11] Hill J. 0., Walking and Type 2 Diabetes, Diabetes Care, 2005, 28 (6), 1524-1525, ISSN 0149-5992.

[12] Kim H. Yang S., Neighborhood Walking and Social Capital: The Correlation between Walking Experience and Individual Perception of Social Capital, Sustainability, 2017, 9(5), ISSN 2071-1050.

[13] Loong C., van Lierop D., El-Geneidy A., On time and ready to go: An analysis of commuters' punctuality and energy levels at work or school, Transportation Research Part F: Traffic Psychology and Behaviour, 2017, 45, 1 - 13, ISSN 1369-8478.

[14] Odyssee-Mure, CHANGE IN DISTANCE TRAVELLED BY CAR, 2019, https://www.odyssee-mure.eu/publications/efficiency-by-sect or/transport/distance-travelled-by-car.html, Accessed: 2019 09-01.

[15] Pal M., Yirgalem M., Anberber M., Giro B., Dasgupta R., Impact of Environmental Pollutionon Animal Health, Journal of Natural History, 2015, 11(1), 1-3, ISSN 0973 - 6166.

[16] Papageorgiou G. Maimaris A., Towards the development of Intelligent Pedestrian Mobility Systems (IPMS), 2017 International Conference on Electrical Engineering and Informatics (ICELTICs), 2017, 251-256.

[17] Papageorgiou G., Maimaris A., Efstathiadou T., Balamou E., EVALUATING ATTITUDES ON THE QUALITY OF SERVICE OF PEDESTRIAN NETWORKS, Ricci S. Brebbia C. A., editors, WIT Transactions on The Built Environment, WIT Press, 2017, 176, 35-41, ISBN 978-1-78466-209-7.
[18] Papageorgiou G., Demetriou D., Balamou E., Maimaris A., Market research and concept study for a smart pedestrian network application, Proceedings of the 11th PErvasive Technologies Related to Assistive Environments Conference, New York, NY, USA, 2018, ACM, PETRA '18, 522-528, ISBN 978-1-4503-6390-7.

[19] Papageorgiou G., Efstathiadou T., Efstathiades A., Maimaris A., Promoting Active Transportation via Information and Communication Technologies, IEEE EUROCON 2019 -18th International Conference on Smart Technologies, 2019, 1-5.

[20] Rogers S., Gardner K., Carlson C., Social capital and walkability as social aspects of sustainability, Sustainability, 2013, 5(8), 3473-3483.

[21] Senauer B. Gemma M., Why is the obesity rate so low in Japan and high in the US? Some possible economic explanations, 2006, Technical report.

[22] The World Bank, Air Pollution Deaths Cost Global Economy US $\$ 225$ Billion, 2016, https://www.worldbank.org/en/news/ press-release/2016/09/08/air-pollution-deaths-cost-globaleconomy-225-billion, Accessed: 2019-09-01.

[23] Wattanapisit A. Thanamee S., Evidence behind 10,000 steps walking, Journal of Health Research, 2017, 31(3), 241-248.

[24] WHO Regional Office for Europe, Data and statistics: The challenge of diabetes, 2014, http://www.euro.who.int/en/healtht opics/noncommunicable-diseases/diabetes/data-and-statist ics, Accessed: 2019-09-01.

[25] WHO Regional Office for Europe, Data and statistics: The challenge of obesity - quick statistics, 2014, http://www.euro.w ho.int/en/health-topics/noncommunicable-diseases/obesity /data-and-statistics, Accessed: 2019-09-01.

[26] World Health Organization, How air pollution is destroying our health, 2019, https://www.who.int/air-pollution/news-andevents/how-air-pollution-is-destroying-our-health, Accessed: 2019-09-01. 